\title{
In Silico Cloning and Chromosomal Localization of EST Sequences that are Related to Leaf Senescence Using Nulli-tetrasomes in Wheat
}

\author{
J.W. GUO ${ }^{1,2, * *}$, Q. LI ${ }^{1 * *}$, W.Q. $\mathrm{CHEN}^{2}$, X. LI ${ }^{1}$, L.Q. LI ${ }^{1}$, T.G. LIU ${ }^{2}$, \\ Z.L. REN ${ }^{1}$ and P.G. LUO ${ }^{1,2, *}$ \\ ${ }^{1}$ Provincial Key Laboratory of Plant Breeding and Genetics, Sichuan Agricultural University, \\ Chengdu, Sichuan 611130, China \\ ${ }^{2}$ State Key Laboratory for Biology of Plant Diseases and Insect Pests, Institute of Plant Protection, \\ Chinese Academy of Agricultural Sciences (CAAS), Beijing 100193, China \\ (Received 10 September 2014; Accepted 9 January 2015; \\ Communicated by M. Molnár-Láng)
}

Leaf senescence is a notably important trait that limits the yield and biomass accumulation of agronomic crops. Therefore, determining the chromosomal position of the expression sequence tags (ESTs) that are associated with leaf senescence is notably interesting in the manipulation of leaf senescence for crop improvement. A total of 32 ESTs that were previously identified during the delaying leaf senescence stage in the stay-green wheat cultivar CN17 were mapped to 42 chromosomes, a chloroplast, a mitochondrion, and a ribosome using in silico mapping. Then, we developed 19 pairs of primers based on these sequences and used them to determine the polymorphisms between the stay-green cultivars (CN12, $\mathrm{CN} 17$, and $\mathrm{CN} 18$ ) and the control cultivar MY11. Among the 19 pairs of primers, 5 pairs produced polymorphisms between the stay-green cultivar and the non-stay-green control. Further studies of Chinese Spring nullisomic-tetrasomics show that JK738991 is mapped to $3 \mathrm{~B}, \mathrm{JK} 738983$ is mapped to $5 \mathrm{D}$, and JK738989 is mapped to 2A, 4A, and 3D. The other two ESTs, JK738994 and JK739003, were not assigned to a chromosome using the Chinese Spring nullisomic-tetrasomics, which indicates that these ESTs may be derived from rye DNA in the wide cross. In particular, the ESTs that produce polymorphisms are notably useful in identifying the stay-green cultivar using molecular marker-assisted selection. The results also suggest that the in silico mapping data, even from a comparison genomic analysis based on the homogeneous comparison, are useful at some points, but the data were not always reliable, which requires further investigation using experimental methods.

Keywords: expression sequence tag (EST), leaf senescence, nullisomic-tetrasomic, in silico mapping, wheat

\footnotetext{
* Corresponding author; E-mail: lpglab@sicau.edu.cn; Phone: +86-28-86290978; Fax: +86-28-86290870

** These authors contributed equally to this work.
} 


\section{Introduction}

Leaf senescence causes a number of cell deaths (Gan and Amasino 1995) in plants, which potentially reduces photosynthesis (Gut et al. 1987); therefore, premature leaf senescence negatively affects the yield stability of rice. During senescence, leaf cells experience dramatic changes in the expression of genes that are involved in photosynthesis, hydrolase activity, oxidoreduction, cellular processes, photorespiration, lipid transport, and biosynthesis pathways (Buchanan-Wollaston et al. 2005; Luo et al. 2013); because of the genetic variations, significant differences in the rate and degree of leaf senescence are observed during the important growth stages (e.g., the grain-filling period) both among species and within species (Thomas and Howarth 2000). Previous studies have reported stay-green phenotypes that result from delayed leaf senescence in many species, which includes several crop species such as wheat (Hortensteiner 2009; Luo et al. 2013). Staygreen cultivars are divided into two principal categories: functional stay-green with photosynthetic activity and cosmetic stay-green without photosynthetic activity (Hortensteiner 2009).

Functional stay-green genotypes have potential significance in crop yield improvement because a delay in the onset or progression of leaf senescence can produce more photosynthetic products from energy sources (e.g., photosynthetic tissues such as leaves) to energy sinks (e.g., developmental organs such as seeds) (Borras et al. 2004). Three wheat cultivars, $\mathrm{CN} 12, \mathrm{CN} 17$, and $\mathrm{CN} 18$, were developed from the progenies of a wheatrye wide cross and are sister lines with a wheat-rye 1BL/1RS translocation (Tang et al. 2008). Many regions in southwest China grow these cultivars because of their high yield (Luo et al. 2009) and good resistance to diseases, including stripe rust (Luo et al. 2008) and powdery mildew (Ren et al. 2009). Previous studies have demonstrated that CN12, $\mathrm{CN} 17$, and CN18 are functional stay-green varieties that maintain high photosynthetic competence during the grain-filling stage (Luo et al. 2006) and are associated with a high yield (Luo et al. 2009). A complete understanding of the underlying molecular mechanism and genetic basis, particularly which genes or chromosome regions are crucial in controlling these traits, is notably important for further use of the varieties in wheat yield improvement.

Further studies on the levels of chlorophylls $a$ and $b$, soluble proteins, and unsaturated fatty acids and on the chloroplast ultrastructure, chloroplast number, and differences in gene expression have provided strong evidence to support that chloroplast ultrastructure regeneration is responsible for the functional stay-green trait of CN17 (Luo et al. 2013). In fact, plants have also evolved sophisticated response mechanisms to adapt to different developmental events during different growth stages and to reprogram gene expression at the transcriptional level (Smart 1994). Therefore, transcript profiling has been widely used to determine how plants transcriptionally respond to developmental events during leaf senescence (Luo et al. 2013). Much progress has been made in elucidating the complex transcriptional regulation mechanism that is responsible for developmental adaption. In a recent study, 32 expression sequence tags (ESTs) were identified in CN17 during the delayed leaf senescence stage; these tags, which were up-regulated in stay-green CN17, 
and showed high homology with known or annotated genes that are associated with senescence-related processes in wheat, rice, barley, pea, and Arabidopsis. Among these ESTs, $31.3 \%$ were directly related to photosynthesis and leaf senescence processes, whereas the other ESTs were indirectly related to these processes (Luo et al. 2013). However, the greatest shortage of this study is the unknown chromosomal locations of the ESTs. Therefore, a large gap remains between the available information and the practice of wheat breeding using molecular marker-assisted selection (MAS).

With the eventual objective of using the stay-green genotypes that were generated by MAS for wheat yield improvement, this study aims to examine the polymorphism of ESTs between stay-green and non-stay-green genotypes, determine the polymorphic ESTs' chromosomal location using nullisomic-tetrasomic, determine whether the polymorphic makers are linked with the 1BL/1RS translocated chromosome, and identify some useful markers for wheat yield improvement by manipulating leaf senescence using MAS.

\section{Materials and Methods}

\section{Plant materials}

The stay-green wheat cultivars CN12, CN17, and CN18 and the non-stay-green wheat cultivar MY11 were used to determine the polymorphism of the ESTs. All 42 Chinese Spring nullisomic-tetrasomic lines were used to determine the chromosomal location of the polymorphic markers, which were kindly provided by Prof. D.C. Liu, Triticeae Research Institute, Sichuan Agricultural University, Chengdu, Sichuan. In addition, the $\mathrm{F}_{2}$ population consisted of $398 \mathrm{~F}_{2}$ individuals, which were genotyped using the corresponding $\mathrm{F}_{2: 3}$ lines that were derived from the cross MY11/CN17, where there were 91 1BL/1RS translocated chromosome homozygotes, 127 1B chromosome homozygotes, and 180 1BL/1RS translocated chromosome and $1 \mathrm{~B}$ chromosome heterozygotes.

\section{In silico mapping of ESTS}

The genomic locations of the wheat ESTs were determined in silico via BLASTN searches against the mapped expressed wheat sequences using Grain Genes 2.0 (http://wheat. pw.usda.gov/GG2/blast.shtml) at a $10^{-5}$ threshold probability and via a BLASTX search against the wheat genome (www.wheatgenome.org). Sequences without a BLASTX hit or those that showed an e-value of less than $10^{-5}$ were excluded from the in silico mapping to the wheat genome and Brachypodium genome. A complete list of the genomic locations of the ESTs is provided in Table $\mathrm{S}^{*}$.

\footnotetext{
* Further details about the Electronic Supplementary Material (ESM) can be found at the end of the article.
} 


\section{Development of the EST-STS markers}

In total, $32 \mathrm{EST}$ sequences that were previously identified in CN17 during the delayed leaf senescence stage (Table S1) were used to develop the STS markers using Primer3 (Rozen and Skaletsky 2000). Then, 19 pairs of EST-STS primers were designed because the other sequences of ESTs were too short to design, and the sequences of these primers are listed in Table S1.

\section{DNA extraction and PCR amplification analysis}

The 5-week-old seedling leaves of all materials were used to extract the total DNA using a previously reported method (Tai and Tanksley 1990). PCR amplification was performed in a PTC-200 thermocycler (MJ Research, Watertown, MA, USA). Each 25- $\mu$ l PCR mixture consisted of forward and reverse primers at concentrations of $200 \mathrm{nmol} / \mathrm{L}, 0.2$ $\mathrm{mmol} / \mathrm{L} \mathrm{dNTPs}, 1.5 \mathrm{mmol} / \mathrm{L} \mathrm{MgCl}_{2}, 1$ unit of Taq polymerase and $60 \mathrm{ng}$ of genomic DNA. PCR was performed using the following program: denaturation at $94{ }^{\circ} \mathrm{C}$ for $3 \mathrm{~min}$, 30 cycles of amplification $\left(30 \mathrm{~s}\right.$ at $94{ }^{\circ} \mathrm{C}, 30 \mathrm{~s}$ at $60{ }^{\circ} \mathrm{C}$, and $30 \mathrm{~s}$ at $72{ }^{\circ} \mathrm{C}$ ) and a final step at $72{ }^{\circ} \mathrm{C}$ for $10 \mathrm{~min}$. Each PCR product was mixed with $3 \mu \mathrm{l}$ of loading buffer $(98 \%$ formamide, $10 \mathrm{mM}$ EDTA [pH 8.0], 0.25\% bromophenol blue and $0.25 \mathrm{mg} / \mathrm{ml}$ xylene cyanol), denatured at $95{ }^{\circ} \mathrm{C}$ for $5 \mathrm{~min}$, and quickly chilled on ice. Subsequently, a 6 - $\mu$ l aliquot of each sample was loaded onto a $6 \%$ polyacrylamide gel (19:1 acrylamide:bisacrylamide, $8 \mathrm{M}$ urea and $1 \times \mathrm{TBE}$ [90 mM Tris-borate ( $\mathrm{pH} 8.3), 2 \mathrm{mM}$ EDTA]) and separated at $80 \mathrm{~W}$ for approximately $1.5 \mathrm{~h}$. Visualization was achieved with silver staining (Bassam et al., 1991).

Table 1. $\chi 2$ analysis of four polymorphic amplicons that were derived from two ESTs associated with leaf senescence

\begin{tabular}{|c|c|c|c|c|c|c|c|c|}
\hline \multirow{2}{*}{ Genotype } & \multicolumn{4}{|c|}{ JK738994 } & \multicolumn{4}{|c|}{ JK739003 A Band } \\
\hline & 0 & 1 & $\chi^{2}$ & $P$ & 0 & 1 & $\chi^{2}$ & $P$ \\
\hline 1B:1B & 31 & 95 & 0.01 & 0.92 & 92 & 35 & 0.44 & 0.51 \\
\hline $1 \mathrm{~B}: 1 \mathrm{~B} / 1 \mathrm{R}$ & 56 & 124 & 3.59 & 0.06 & 123 & 57 & 3.92 & 0.05 \\
\hline 1B/1R:1B/1R & 21 & 70 & 0.18 & 0.67 & 66 & 25 & 0.30 & 0.59 \\
\hline Total & 108 & 289 & 1.03 & 0.31 & 281 & 117 & 4.10 & 0.04 \\
\hline \multirow{2}{*}{ Genotype } & \multicolumn{4}{|c|}{ JK739003 B Band } & \multicolumn{4}{|c|}{ JK739003 C Band } \\
\hline & 0 & 1 & $\chi^{2}$ & $P$ & 0 & 1 & $\chi^{2}$ & $P$ \\
\hline 1B:1B & 32 & 95 & 0.00 & 0.96 & 35 & 92 & 0.44 & 0.51 \\
\hline $1 \mathrm{~B}: 1 \mathrm{~B} / 1 \mathrm{R}$ & 33 & 147 & 4.27 & 0.04 & 50 & 130 & 0.74 & 0.39 \\
\hline 1B/1R:1B/1R & 27 & 64 & 1.06 & 0.30 & 28 & 63 & 1.62 & 0,20 \\
\hline Total & 92 & 306 & 0.75 & 0.39 & 113 & 285 & 2.44 & 0.12 \\
\hline
\end{tabular}

Notes: 0 denotes the polymorphic amplicon that is identical to that of the non-stay-green genotype control MY11; 1 denotes the polymorphic amplicons that are identical to that of stay-green genotype CN17. 


\section{Statistical analysis and linkage analysis}

Chi-squared tests were performed to determine the goodness-of-fit of the segregation data with the hypothesized 3:1 ratios for the marker products in different translocation genotypes of $\mathrm{F}_{2: 3}$ lines using the SigmaPlot 2001 software (SPSS Inc., Chicago, IL, USA). The linkage relationship between the EST-STS markers and 1BL/1RS translocated chromosome was determined using JoinMap 4 (Wageningen, Netherlands). In total, $398 \mathrm{~F}_{2: 3}$ lines that were derived from MY11/CN17 (Table 1) were used to determine the linkage relationship using JoinMap 4 with an LOD threshold of 3.0.

\section{Results}

\section{In silico mapping}

The genomic locations of the wheat ESTs that are associated with leaf senescence were determined in silico using BLASTN searches. The results (Table S1) demonstrate that 31 $(96.9 \%)$ of the 32 ESTs have homogeneous sequences in the publically available data, whereas only 1 (3.1\%) does not have a homogeneous sequence (Luo et al. 2013). Among the 31 ESTs with homogeneous sequences, 11 were assigned to a unique chromosome; 7 were assigned to multiple chromosomes, where JK738986, which encodes a putative leaf senescence-associated protein, has a homogeneous sequence on every chromosome; 7 were assigned to a chloroplast; 5 were assigned to a ribosome; and only 1 was assigned to a mitochondrion (Table S1).

\section{Polymorphism of the ESTs between stay-green and non-stay-green cultivars}

We only developed 19 pairs of EST-STS primers according to the sequences of 19 of 32 ESTs that were associated with leaf senescence because the other EST sequences were too short to design primers (Table S1). The PCR amplification results indicate that every primer pair produces clear products (Table S1), which suggests that the primers work notably well. Further analysis finds that the primers of 5 ESTs (JK738991, JK738983, JK738989, JK738994, and JK739003) produce polymorphisms between stay-green and non-stay-green wheat genotypes (Fig. 1 and Table S1). In addition, the products of the polymorphic markers were identical in three stay-green genotypes $\mathrm{CN} 12, \mathrm{CN} 17$, and CN18 (Fig. 1).

\section{Chromosomal location of the polymorphic EST-STS markers}

Further study of 5 polymorphic markers using Chinese Spring nullisomic-tetrasomic lines indicates that 3 ESTs were located on specific chromosomes (Table S1). JK738991, which was assigned to $1 \mathrm{~A}$ using in silico mapping, was localized to chromosome $3 \mathrm{~B}$, whereas JK38983, which was assigned to multiple chromosomes such as 6A, 6B, and 6D, was localized to the unique chromosome 5D. In contrast, JK738989, which was assigned to the unique chromosome $2 \mathrm{~B}$ using in silico mapping, produces three products, 


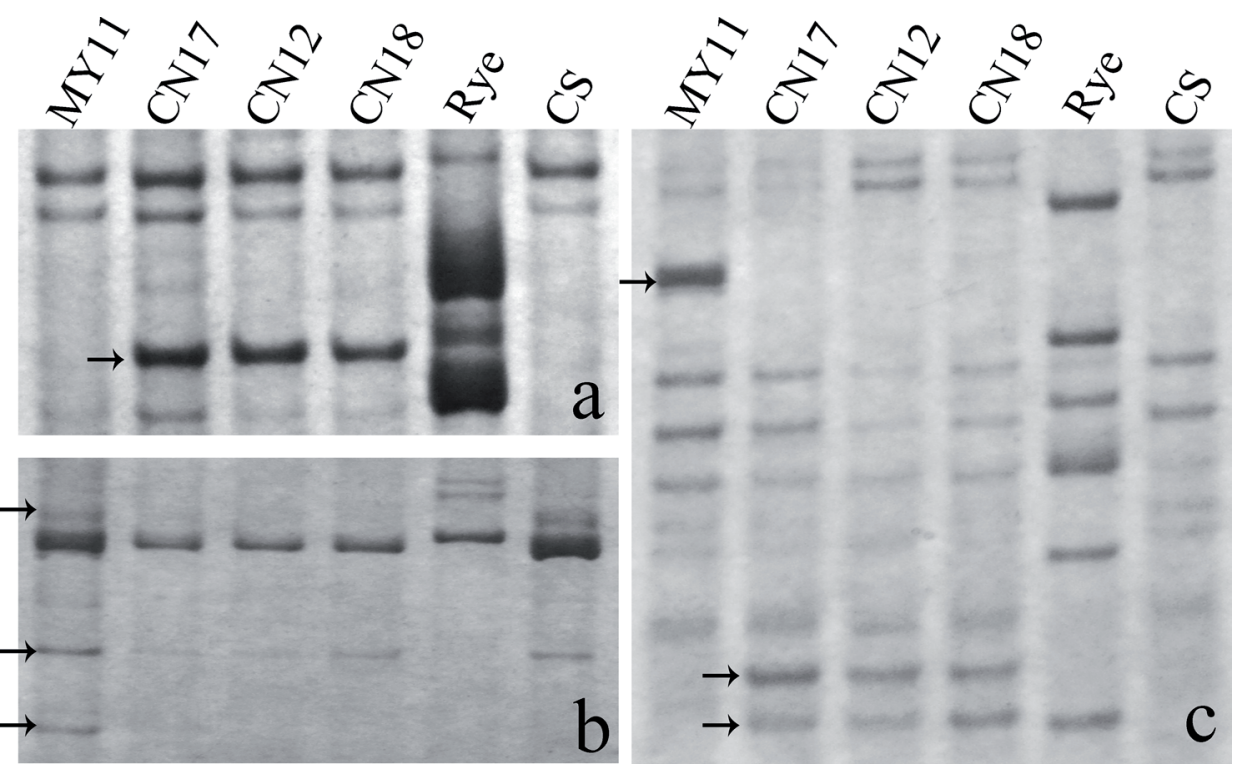

Figure 1. Polymorphic analysis of ESTs associated with leaf senescence among wheat stay-green genotypes (CN12, CN17, and CN18) with 1BL/1RS translocated chromosome and wheat non-stay-green genotypes (MY11, and CS) without the translocated chromosome. CS: Chinese Spring; a: the primer derived from JK738994; b: the primer derived from JK738989; c: the primer derived from JK739003

JK738989-A, JK738989-B, and JK738989-C, which were located at 2A, 4A, and 3D, respectively. The other polymorphic ESTs (JK738994 and JK739003), which were assigned to chromosomes $2 \mathrm{~A}$ and $2 \mathrm{~B}$ and a ribosome, could not be located on any specific chromosome using the Chinese Spring nullisomic-tetrasomic lines.

\section{Independent assortment of JK738994, JK739003, and the 1BL/1RS} translocated chromosome

There are polymorphisms in both JK738994 and JK739003 between stay-green and nonstay-green genotypes, but the chromosomal location was not determined using the nullisomic-tetrasomic lines. These results indicate that these ESTs may be on the 1BL/1RS translocated chromosome as a rye genomic component. A linkage analysis among JK738994, JK739003, and the 1BL/1RS translocated chromosome using the $\mathrm{F}_{2: 3}$ lines that were derived from MY11/CN17 was performed, and the results (Fig. S1 and Table 1) indicate that JK738994, JK739003, and the 1BL/1RS translocated chromosome assort independently of one another. Moreover, the three different products (JK739003-A, JK739003-B, and JK739003-C) that were produced by the identical primers of JK739003 are not linked to each other in separate populations (Table 1). In addition, almost all prod- 
ucts except JK739003-A exhibit a 3-fold existence: 1 null ratio at $P=0.05$ level in the separating populations, although JK739003-A has a distorted separation in the separating population at $P=0.04$, which is almost 0.05 (Table 1).

\section{Discussion}

As an important trait, leaf senescence affects the yield and biomass accumulation of agronomic crops (Guo and Gan 2014), and significant progression in the understanding of leaf senescence has enabled the improvement of wheat yield by manipulating the onset or progression of leaf senescence (Luo et al. 2013). The genetic variability of the duration of leaf senescence during grain filling positively affects wheat grain yields (Bogard et al. 2011). Delaying leaf senescence, which is also called stay-green, has practically been achieved in wheat breeding; three stay-green wheat cultivars were developed and released in 2003 by the wheat-breeding group led by Z.L. Ren at Sichuan Agricultural University and have been widely cultivated in Southwestern China because of their high yield (Ren et al. 2003).

The availability of ESTs that are associated with leaf senescence in CN17 is useful to improve the wheat yield (Luo et al. 2013). To make these ESTs maneuverable in wheat breeding using MAS, determining the chromosomal location is notably important. In this study, 31 of 32 ESTs that were related to leaf senescence were assigned to different genomic or chromosomal locations based on an in silico homology analysis (Table S1). We further determined the chromosomal location of 5 polymorphic ESTs (Table S1), and there were complete differences in the genomic and chromosomal locations using the Chinese Spring nullisomic-tetrasomic lines compared to those using in silico mapping. However, in the past, ESTs were believed to be conserved portions of expression genes, which are used as the theoretical basis for comparative genomics analyses (Liu et al. 2012). This result warns plant breeders and researchers that the homology analysis and comparative genomics analysis results are not reliable.

The polymorphism of the ESTs between stay-green genotypes and the control shows a true difference in the DNA levels in the chromosomal region that produces the ESTs. In addition, the polymorphic ESTs and the corresponding polymorphic EST-STS markers are notably useful in wheat yield improvement using MAS. We previously identified 5 polymorphic EST-STS markers among 19 EST-STS markers that were derived from 19 ESTs (Table S1), which constitutes $26.3 \%$ polymorphic ESTs. This result indicates a low false positive rate in the ESTs when SSH methods are used (Diatchenko et al. 1996) because these ESTs were detected using an SSH cDNA library (Luo et al. 2013). In addition, the five ESTs, JK738991, JK738983, JK738989, JK738994, and JK739003, exhibit polymorphisms between the stay-green genotypes and the control (Fig. 1 and Table S1), which indicates that there are differences in the chromosomal region, which possibly has a greater contribution in delaying the leaf senescence during the grain-filling stage; therefore, EST-STSs perhaps play an important role in wheat breeding using MAS. 
The EST JK738994 was assigned in silico to chromosomes 2A and 2B (Table S1), whereas its true chromosomal location was not determined using the Chinese Spring nullisomic-tetrasomic lines (Table S1). Although the detailed chromosomal location remains unknown, the fact that JK738994 took on a 3 dominant/ 1 recessive $\left(\chi^{2}=1.03\right.$ and $P=0.31$ ) ratio as a Mendelian genetic factor in the separating population (Table 1) suggests that this EST is located in a nuclear genomic DNA instead of a cytoplasmic genomic DNA. The EST JK739003 was assigned in silico to a ribosome, and its specific location was not determined using the Chinese Spring nullisomic-tetrasomic lines (Table S1). Both products (JK739003-B, and JK739003-C) consistently exhibit the genetic rule of a single Mendelian factor in the separating population (Table 1), and the $P$ value for JK739003-A separation is 0.04 , or nearly 0.05 . This result strongly suggests that JK 739003 is located on nuclear genomic DNA instead of cytoplasmic genomic DNA.

We were interested in JK738994 and JK739003 because they were not located on a specific chromosome using the Chinese Spring nullisomic-tetrasomic lines; therefore, they may be on the $1 \mathrm{BL} / \mathrm{RS}$ translocated chromosome or the notably small chromosomal segments that were integrated into the wheat genome using cryptic translocation. However, further linkage analysis of JK738994, JK739003-A, JK739003-B, and JK739003-C show that these ESTs assort independently of one another (Table 1), which excludes the possibility that all of them are on the $1 \mathrm{BL} / \mathrm{RS}$ translocated chromosome. Therefore, we accept the potential possibility that most of them are separately located on the putative rye-derived chromosome segment in the nuclear genome of the stay-green genotypes.

Comprehensively, the results suggest that plant breeders should use more caution when they use the information from homology analyses and comparative genomics and that the polymorphic EST-STS markers are more useful during wheat breeding with MAS. Therefore, we will further determine the chromosomal location of both JK738994 and JK739003 and elucidate the relationship between the polymorphic EST-STS markers and the stay-green phenotype.

\section{Acknowledgements}

Financial support was provided by the National Natural Science Foundation of China (31271721), the Provincial Science and Technology Foundation for Young Scientists of Sichuan China (2010JQ0042), and the State Key Laboratory for Biology of Plant Disease and Insect Pests, China (SKLOF201410).

\section{References}

Bassam, B.J., Caetano-Anolles, G., Gresshoff, P.M. 1991. Fast and sensitive silver staining of DNA in polyacrylamide gels. Anal. Biochem. 196:80-83.

Bogard, M., Jourdan, M., Allard, V., Martre, P., Perretant, M.R., Ravel, C., Heumez, E., Ordord, S., Snape, J., Griffiths, S., Gaju, O., Foulkes, J., Gouis, J.L. 2011. Anthesis date mainly explained correlations between post-anthesis leaf senescence, grain yield, and grain protein concentration in a winter wheat population segregating for flowering time QTLs. J. Exp. Bot. 62:3621-3636.

Borras, L., Slafer, G.A., Otegui, M.E. 2004. Seed dry weight response to source-sink manipulations in wheat, maize and soybean: a quantitative reappraisal. Field Crops Res. 86:131-146. 
Buchanan-Wollaston, V., Page, T., Harrison, E., Breeze, E., Lim, P.O., Nam, H.G., Lin, J.F., Wu, S.H., Swidzinski, J., Ishizaki, K., Leaver, C.J. 2005. Comparative transcriptome analysis reveals significant differences in gene expression and signaling pathways between developmental and dark/starvation-induced senescence in Arabidopsis. Plant J. 42:567-585.

Diatchenko, L., Lau, Y.F.C., Campbell, A.P., Chenchik, A., Moqadam, F., Huang, B., Lukyanov, S., Lukyanov, K., Gurskaya, N., Sverdlov, E.D. 1996. Suppression subtractive hybridization: a method for generating differentially regulated or tissue-specific cDNA probes and libraries. Proc. Natl Acad. Sci. USA 93:60256030 .

Gan, S., Amasino, R.M. 1995. Inhibition of leaf senescence by autoregulated production of cytokinin. Science 270:1986-1988.

Guo, Y.F., Gan, S.S. 2014. Translational researches on leaf senescence for enhancing plant productivity and quality. J. Exp. Bot. doi:10.1093/jxb/eru248

Gut, H., Ruts, C., Matile, P., Thomas, H. 1987. Leaf senescence in a non-yellowing mutant of Festuca pratensis: degradation of carotenoids. Physiologica Plant. 70:659-663.

Hortensteiner, S. 2009. Stay-green regulates chlorophyll and chlorophyll-binding protein degradation during senescence. Trend. Plant Sci. 14:155-162.

Liu, Z., Zhu, J., Cui, Y., Liang, Y., Wu, H., Song, W., Liu, Q., Yang, T., Sun, Q., Liu, Z. 2012. Identification and comparative mapping of a powdery mildew resistance gene derived from wild emmer (Triticum turgidum var. dicoccoides) on chromosome 2BS. Theor. Appl. Genet. 124:1041-1049.

Luo, L.P., Ren, Z.L., Wu, X.H., Zhang, H.Y., Zhang, H.Q., Feng, J. 2006. Structural and biochemical mechanism responsible for the stay-green phenotypes in common wheat. Chinese Sci. Bull. 51:2595-2603.

Luo, P.G., Zhang, H.Y., Shu, K., Zhang, H.Q., Luo, H.Y., Ren, Z.L. 2008. Stripe rust (Puccinia striiformis f. sp. tritici) resistance in wheat with the wheat-rye 1BL/1RS chromosomal translocation. Canadian J. Plant Path. 30:254-259.

Luo, P.G., Zhang, H.Y., Shu, K., Wu, X.H., Zhang, H.Q., Ren, Z.L. 2009. The physiological genetic effects of 1BL/1RS translocated chromosome in 'stay green' wheat cultivars CN17. Canadian J. Plant Sci. 89:1-10.

Luo, P.G., Deng, K.J., Hu, X.Y., Li, L.Q., Li, X., Chen, J.B., Zhang, H.Y., Tang, Z.X., Zhang, Y., Sun, Q.X., Tan, F.Q., Ren, Z.L. 2013. Chloroplast ultrastructure regeneration with protection of photosystem II is responsible for the functional 'stay-green' trait in wheat. Plant Cell Environ. 36:683-696.

Rozen, S., Skaletsky, H. 2000. Primer3 on the WWW for general users and for biologist programmers. Methods Mol. Biol. 132:365-386.

Ren, Z.L., He, Z.C., Zhang, H.Q., Tan, F.Q., Jiang, H.R. 2003. A new record on wheat yield in Chengdu plain with consonance type cultivar chuan-nong 17. J. Sichuan Agric. Univ. 21:85-87.

Ren, T.H., Yang, Z.J., Yan, B.J., Zhang, H.Q., Fu, S.L., Ren, Z.L. 2009. Development and characterization of a new 1BL.1RS translocation line with resistance to stripe rust and powdery mildew of wheat. Euphytica 169:207-213.

Smart, C.M. 1994. Gene expression during leaf senescence. New Phytol. 126:419-448.

Tai, T.H., Tanksley, S.D. 1990. A rapid and inexpensive method for isolation of total DNA from dehydrated plant tissue. Plant Mol. Biol. Rep. 8:297-303.

Tang, Z.X., Fu, S.L., Ren, Z.L., Zhou, J.P., Yan, B.J., Zhang, H.Q. 2008. Variation of tandem repeat regulatory element and promoter regions revealed by wheat-rye amphiploids. Genome 51:399-408.

Thomas, H., Howarth, C.J. 2000. Five ways to stay green. J. Exp. Bot. 51:329-337. 


\section{Electronic Supplementary Material (ESM)}

Electronic Supplementary Material (ESM) associated with this article can be found at the website of CRC at http://www.akademiai.com/content/120427/

Electronic Supplementary Table S1. Chromosomal assignment of EST associated with leaf senescence using both in silico and Chinese Spring nullisomic-tetrasomic methods

Electronic Supplemenary Figure S1. Linkage analysis of polymorphic amplicons with wheat-rye 1BL/1RS translocated chromosome in the $\mathrm{F}_{2}$ population

$\mathrm{CN} 12, \mathrm{CN} 17$, and CN18 are wheat stay-green genotypes with 1BL/1RS translocated chromosome, whereas CS (Chinese Spring) and MY11 are wheat non-stay-green genotypes without the translocated chromosome. a denotes the primer derived from JK738994; $b$ denotes the primer derived from JK739003; 1, 8, 10, 12, 13, 15, $20,21,22,24$, and 27 for $\mathrm{F}_{2}$ individuals with homozygotes of wheat chromosome 1B; $2,3,4,5,6,7,11,14$, $16,17,18$, and 25 for $\mathrm{F}_{2}$ individuals with heterozygotes of wheat chromosome $1 \mathrm{~B}$ and wheat-rye translocated chromosome $1 \mathrm{BL} / 1 \mathrm{RS} ; 9,19,23,26$, and 28 for $\mathrm{F}_{2}$ individuals with homozygotes with wheat-rye translocated chromosome $1 \mathrm{BL} / 1 \mathrm{RS}$. 